\title{
ON THE BESOV REGULARITY OF THE BIFRACTIONAL BROWNIAN MOTION
}

\author{
BY
}

\author{
BRAHIM BOUFOUSSI (MARRAKESH) AND YASSINE NACHIT (MARRAKESH)
}

\begin{abstract}
Our aim is to improve Hölder continuity results for the bifractional Brownian motion $(\mathrm{bBm})\left(B^{\alpha, \beta}(t)\right)_{t \in[0,1]}$ with $0<\alpha<1$ and $0<\beta \leqslant 1$. We prove that almost all paths of the $\mathrm{bBm}$ belong to (resp. do not belong to) the $\operatorname{Besov}$ spaces $\operatorname{Bes}(\alpha \beta, p)(\operatorname{resp} . \operatorname{bes}(\alpha \beta, p)$ ) for any $\frac{1}{\alpha \beta}<p<\infty$, where bes $(\alpha \beta, p)$ is a separable subspace of $\operatorname{Bes}(\alpha \beta, p)$. We also show similar regularity results in the Besov-Orlicz space $\operatorname{Bes}\left(\alpha \beta, M_{2}\right)$ with $M_{2}(x)=e^{x^{2}}-1$. We conclude by proving the Itô-Nisio theorem for the bBm with $\alpha \beta>1 / 2$ in the Hölder spaces $\mathcal{C}^{\gamma}$ with $\gamma<\alpha \beta$.
\end{abstract}

2020 Mathematics Subject Classification: Primary 60G15; Secondary 60G18, 60G17.

Key words and phrases: bifractional Brownian motion, self-similar, Besov spaces, Besov-Orlicz spaces, Itô-Nisio.

\section{INTRODUCTION}

Let $\left(B^{\alpha, \beta}(t)\right)_{t \geqslant 0}$ be a bifractional Brownian motion (bBm for short), i.e., a centred real-valued Gaussian process with covariance function

$$
R^{\alpha, \beta}(s, t):=R(s, t)=\frac{1}{2^{\beta}}\left(\left(t^{2 \alpha}+s^{2 \alpha}\right)^{\beta}-|t-s|^{2 \alpha \beta}\right),
$$

where $\alpha \in(0,1)$ and $\beta \in(0,1]$. Observe that when $\beta=1, B^{\alpha, 1}$ is a fractional Brownian motion with Hurst parameter $\alpha \in(0,1)$. However, the increments of $B^{\alpha, \beta}$ are not stationary except for $\beta=1$. The $\mathrm{bBm}$ has the following general properties: it is self-similar with index $\alpha \beta$, that is, for every $a>0$,

$$
\left\{B^{\alpha, \beta}(a t), t \geqslant 0\right\} \stackrel{d}{=}\left\{a^{\alpha \beta} B^{\alpha, \beta}(t), t \geqslant 0\right\},
$$

where $X \stackrel{d}{=} Y$ means that the two processes have the same finite-dimensional distributions. It is a quasi-helix (see [17] and [18] for various properties and appli- 
cations of quasi-helices) since for every $s, t \in[0, T]$, we have

$$
2^{-\beta}|t-s|^{2 \alpha \beta} \leqslant \mathbb{E}\left(B^{\alpha, \beta}(t)-B^{\alpha, \beta}(s)\right)^{2} \leqslant 2^{1-\beta}|t-s|^{2 \alpha \beta} .
$$

Based on the fractional Brownian motion structure, Houdré and Villa [14] have constructed the bifractional Brownian motion as a more general self-similar Gaussian process. Russo and Tudor [27] have shown that the $\mathrm{bBm}$ behaves, in terms of sample path properties, like a fractional Brownian motion with Hurst parameter $\alpha \beta$ (one can see that clearly in Lei and Nualart decomposition). There is a rich literature investigating the properties of the bifractional Brownian motion; we refer for example to the following non-exhaustive list: Bojdecki et al. [4], El-Nouty [10], El-Nouty and Journé [11], Kruk et al. [20], Es-Sebaiy and Tudor [12], Tudor and Xiao [30], Bardina and Es-Sebaiy [3] and Lei and Nualart [22], to mention but a few. In this last paper the following decomposition of the $\mathrm{bBm}$ was essentially shown:

$$
\left\{C_{2} B^{\alpha \beta}(t), t \geqslant 0\right\} \stackrel{d}{=}\left\{C_{1} X^{\alpha, \beta}(t)+B^{\alpha, \beta}(t), t \geqslant 0\right\},
$$

where $C_{1}, C_{2}$ are constants, $\left(B^{\alpha \beta}(t)\right)_{t \geqslant 0}$ is a fractional Brownian motion (fBm) with parameter $\alpha \beta$ and $\left(X^{\alpha, \beta}(t)\right)_{t \geqslant 0}$ is a Gaussian process with infinitely differentiable trajectories on $(0, \infty)$ and absolutely continuous on $[0, \infty)$. On the other hand, we know from Ciesielski et al. [9] that almost all paths of the fBm $\left(B^{\alpha \beta}(t)\right)_{t \geqslant 0}$ belong (resp. do not belong) to the Besov spaces $\operatorname{Bes}(\alpha \beta, p)$ (resp. to the separable subspaces bes $(\alpha \beta, p)$ ). In fact, a stronger regularity result was obtained in the Besov-Orlicz space $\operatorname{Bes}\left(\alpha \beta, M_{2}\right)$, where $M_{2}(x)=e^{x^{2}}-1$, (definitions are given in Section 2). Needless to mention that if we take $0<a<b$ one can deduce directly by Lei and Nualart decomposition that the sample paths of $\left(B^{\alpha, \beta}(t)\right)_{a \leqslant t \leqslant b}$ have the same Besov regularity as those of fractional Brownian motion of parameter $\alpha \beta$. Otherwise, we are unable to get the Hölder regularity of $X^{\alpha, \beta}$ on intervals of type $[0, \varepsilon]$ for $\varepsilon>0$, since the trajectories of this process are only absolutely continuous near 0 . Hence we cannot derive directly from Lei and Nualart decomposition the Besov regularity for the bBm on the interval $[0, \varepsilon]$. Our main purpose in this paper is to investigate the Besov regularity for sample paths of the $\mathrm{bBm}\left(B^{\alpha, \beta}(t)\right)$ for $t \in[0,1]$.

The Besov spaces $\operatorname{Bes}(\gamma, p)$ are a general framework to investigate the modulus of smoothness in $L^{p}$-norms for trajectories of continuous time stochastic processes. In our paper we are concerned with a particular class of Besov spaces of real functions $(f(t), t \in[0,1])$ (for a more general context see Triebel [29]). One can get an improvement of the regularity in Besov spaces $\operatorname{Bes}(\gamma, p)$ by using the Besov-Orlicz spaces $\operatorname{Bes}\left(\gamma, M_{2}\right)$, because of the following injections: for all $p \geqslant 1$,

$$
\operatorname{Bes}\left(\gamma, M_{2}\right) \hookrightarrow \operatorname{Bes}(\gamma, p)
$$

We denote by $\mathcal{C}^{\gamma}$ the space of functions satisfying the Hölder condition of order $\gamma>0$, endowed with the usual norm. It is known that Besov spaces cover Hölder 
spaces as particular cases, more precisely $\mathcal{C}^{\gamma}=\operatorname{Bes}(\gamma, \infty)$. In addition, for any $\varepsilon>0$ and $p$ large enough, we have the following continuous injections (see Section 2):

$$
\mathcal{C}^{\gamma+\varepsilon}(I) \hookrightarrow \operatorname{bes}(\gamma, p)(I) \quad \text { and } \quad \mathcal{C}^{\gamma}(I) \hookrightarrow \operatorname{Bes}(\gamma, p)(I) \hookrightarrow \mathcal{C}^{\gamma-1 / p}(I),
$$

where $\operatorname{bes}(\gamma, p)$ is a separable subspace of $\operatorname{Bes}(\gamma, p)$. It is well known that almost surely the sample paths of the $\mathrm{bBm}\left(B^{\alpha, \beta}(t)\right)_{t \geqslant 0}$ belong to the Hölder space $\mathcal{C}^{\gamma}$ for $\gamma<\alpha \beta$, and do not belong a.s. to $\mathcal{C}^{\alpha \beta}$.

Our aim is to improve these classical results by showing that we can get smoothness of order $\alpha \beta$ in the $\operatorname{Besov}$ space $\operatorname{Bes}(\alpha \beta, p)$ for $\frac{1}{\alpha \beta}<p<\infty$, or even in the Besov-Orlicz space $\operatorname{Bes}\left(\alpha \beta, M_{2}\right)$. This is the best regularity one can get in the context of Besov spaces, because we also prove that almost surely the trajectories of the bBm do not belong to the separable spaces bes $(\alpha \beta, p)$ for $\frac{1}{\alpha \beta}<p<\infty$. So the above injections explain clearly the sharpness of our results. Note that our paper leads to some previous Besov regularity results: When $\alpha=1 / 2$ and $\beta=1$ we get the standard Brownian motion considered in [7] and [8], for $\beta=1$ we recover the $\mathrm{fBm}$ situation considered in [9], and the case $\alpha=\beta=1 / 2$ corresponds to the regularity of the mild solution for a linear stochastic heat equation driven by a white noise (see [6]).

Among Itô's accomplishments, there is the Itô-Nisio theorem (cf. [16]), in which the authors have established on the one hand a general improvement of the Fourier series decomposition of the Brownian motion, and on the other hand a generalization of Wiener's construction of the Brownian motion. They gave the expansion as the convergence of normalized sums of independent random variables. Later, Kerkyacharian and Roynette [19] proved the same result of the Itô-Nisio in Hölder spaces with a sample proof. In this paper we show the Itô-Nisio theorem for the bBm with $\alpha \beta>1 / 2$ in the Hölder spaces $\mathcal{C}^{\gamma}$ with $\gamma<\alpha \beta$. The case $\beta=1$ corresponds to the $\mathrm{fBm}$ with Hurst parameter $\alpha>1 / 2$.

This paper is organized as follows. In the second section we give a brief introduction to Besov spaces. The third section is devoted to the study of Besov regularity for sample paths of the bifractional Brownian motion. In the fourth section we investigate the Itô-Nisio theorem for $\mathrm{bBm}$ with $\alpha \beta>1 / 2$. The proofs of our results use technical and fine calculations based on dyadic coordinate expansions of the bifractional Brownian motion and descriptions of Besov norms in terms of the corresponding expansion coefficients of a function.

\section{PRELIMINARIES}

2.1. Besov spaces. Let $I \subset \mathbb{R}$ be a compact interval, $1 \leqslant p<\infty$ and $f \in L^{p}(I ; \mathbb{R})$. For any $t>0$ we define

$$
\Delta_{p}(f, I)(t)=\sup _{|s| \leqslant t}\left\{\int_{I_{s}}|f(x+s)-f(x)|^{p} d x\right\}^{1 / p},
$$


where $I_{s}=\{x \in I ; x+s \in I\} . \Delta_{p}(f, I)(t)$ denotes the modulus of continuity of $f$ in the $L^{p}$-norm. For $\gamma>0$, we consider the norm

$$
\|f\|_{\gamma, p}:=\|f\|_{L^{p}(I)}+\sup _{0<t \leqslant 1} \frac{\Delta_{p}(f, I)(t)}{t^{\gamma}} .
$$

The Besov space is given by $\operatorname{Bes}(\gamma, p)(I)=\left\{f \in L^{p}(I) ;\|f\|_{\gamma, p}<\infty\right\}$. The space $\left(\operatorname{Bes}(\gamma, p)(I),\|\cdot\|_{\gamma, p}\right)$ is a non-separable Banach space. We also define $\operatorname{bes}(\gamma, p)(I)=\left\{f \in L^{p}(I) ; \Delta_{p}(f, I)(t)=o\left(t^{\gamma}\right)\right.$ as $\left.t \rightarrow 0^{+}\right\}$, a separable subspace of $\operatorname{Bes}(\gamma, p)(I)$. For $p=\infty$, the space $\operatorname{Bes}(\gamma, \infty)(I)$ is defined in the same way by using the usual $L^{\infty}$-norm.

In the case of the unit interval $I=[0,1]$ Besov spaces are characterized in terms of sequences of the coefficients of the expansion of continuous functions with respect to the Schauder basis. The following isomorphism theorem was established by Ciesielski [7] (see also Ciesielski et al. [9]):

THEOREM 2.1. Let $1<p<\infty$ and $1 / p<\gamma<1$.

(1) $\operatorname{Bes}(\gamma, p)([0,1])$ is linearly isomorphic to a sequence space and we have the following equivalence of norms:

$$
\|f\|_{\gamma, p} \sim \max \left\{\left|f_{0}\right|,\left|f_{1}\right|, \sup _{j} 2^{-j(1 / 2-\gamma+1 / p)}\left[\sum_{k=1}^{2^{j}}\left|f_{j k}\right|^{p}\right]^{1 / p}\right\},
$$

where the coefficients $\left\{f_{0}, f_{1}, f_{j k}, j \geqslant 0,1 \leqslant k \leqslant 2^{j}\right\}$ are given by

$$
\begin{gathered}
f_{0}=f(0), \quad f_{1}=f(1)-f(0), \\
f_{j k}=2 \cdot 2^{j / 2}\left\{f\left(\frac{2 k-1}{2^{j+1}}\right)-\frac{1}{2} f\left(\frac{2 k}{2^{j+1}}\right)-\frac{1}{2} f\left(\frac{2 k-2}{2^{j+1}}\right)\right\} .
\end{gathered}
$$

(2) $f$ is in $\operatorname{bes}(\gamma, p)([0,1])$ if and only if

$$
\lim _{j \rightarrow \infty} 2^{-j(1 / 2-\gamma+1 / p)}\left[\sum_{k=1}^{2^{j}}\left|f_{j k}\right|^{p}\right]^{1 / p}=0 .
$$

2.2. Besov-Orlicz spaces. Let $I \subset \mathbb{R}$ be a compact interval, $\gamma \in(0,1)$, and $M_{2}$ the Young function defined by $M_{2}(x)=e^{x^{2}}-1$. The Orlicz space $L_{M_{2}}(I)$ is the space of measurable functions $f: I \rightarrow \mathbb{R}$ such that

$$
\|f\|_{M_{2}}^{*}:=\inf _{\lambda>0} \frac{1}{\lambda}\left[1+\int_{I} M_{2}(\lambda f(t)) d t\right]<\infty .
$$

It is more suitable to use an equivalent norm to $\|\cdot\|_{M_{2}}^{*}$ (see Fernique [13] or Ciesielski [8] for a proof):

$$
\|f\|_{M_{2}}=\sup _{p \geqslant 1} \frac{\|f\|_{L^{p}(I)}}{\sqrt{p}} .
$$


Let $\Delta_{M_{2}}(f, I)(t)$ be the modulus of continuity of $f$ in the Orlicz space $L_{M_{2}}(I)$ defined as

$$
\Delta_{M_{2}}(f, I)(t)=\sup _{p \geqslant 1} \frac{\Delta_{p}(f, I)(t)}{\sqrt{p}} .
$$

For $\gamma>0$, we consider the norm

$$
\|f\|_{\gamma, M_{2}}=\|f\|_{M_{2}}+\sup _{0<t \leqslant 1} \frac{\Delta_{M_{2}}(f, I)(t)}{t^{\gamma}} .
$$

The Besov-Orlicz space is defined by

$$
\operatorname{Bes}\left(\gamma, M_{2}\right)(I):=\left\{f \in L_{M_{2}}(I) ;\|f\|_{\gamma, M_{2}}<\infty\right\} .
$$

$\operatorname{Bes}\left(\gamma, M_{2}\right)(I)$ endowed with the norm $\|\cdot\|_{\gamma, M_{2}}$ is a non-separable Banach space. We introduce bes $\left(\gamma, M_{2}\right)(I)=\left\{f \in L_{M_{2}}(I) ; \Delta_{M_{2}}(f, I)(t)=o\left(t^{\gamma}\right)\right.$ as $\left.t \rightarrow 0^{+}\right\}$, a separable subspace of $\operatorname{Bes}\left(\gamma, M_{2}\right)(I)$.

With the same notations as in Theorem 2.1, we have the following characterization of Besov-Orlicz spaces (see Ciesielski [8] or Ciesielski et al. [9]):

THEOREM 2.2.

(1) $\operatorname{Bes}\left(\gamma, M_{2}\right)([0,1])$ is linearly isomorphic to a sequence space and we have the following equivalence of norms:

$$
\|f\|_{\gamma, M_{2}} \sim \max \left\{\left|f_{0}\right|,\left|f_{1}\right|, \sup _{p, j} \frac{1}{\sqrt{p}} 2^{-j(1 / 2-\gamma+1 / p)}\left[\sum_{k=1}^{2^{j}}\left|f_{j k}\right|^{p}\right]^{1 / p}\right\},
$$

(2) $f$ belongs to $\operatorname{bes}\left(\gamma, M_{2}\right)([0,1])$ if and only if

$$
\lim _{j \rightarrow \infty} \sup _{p \geqslant 1} \frac{1}{\sqrt{p}} 2^{-j(1 / 2-\gamma+1 / p)}\left[\sum_{k=1}^{2^{j}}\left|f_{j k}\right|^{p}\right]^{1 / p}=0 .
$$

REMARK 2.3. (1) Let $1 \leqslant p<\infty$ and $0<\gamma<\gamma^{\prime}<1$. Then

$$
\operatorname{Bes}\left(\gamma^{\prime}, p\right)(I) \hookrightarrow \operatorname{bes}(\gamma, p)(I) .
$$

(2) We denote by $\mathcal{C}^{\gamma}(I)$ the Hölder space defined by

$$
\mathcal{C}^{\gamma}(I):=\left\{f \in C(I) ; \sup _{\substack{x, y \in I \\ x \neq y}} \frac{|f(x)-f(y)|}{|x-y|^{\gamma}}<\infty\right\},
$$

endowed with the norm

$$
\|f\|_{\gamma}=\sup _{x \in I}|f(x)|+\sup _{\substack{x, y \in I \\ x \neq y}} \frac{|f(x)-f(y)|}{|x-y|^{\gamma}} .
$$


It has the following properties:

- $\operatorname{Bes}(\gamma, \infty)(I)=\mathcal{C}^{\gamma}(I)$.

- For $1 \leqslant p<\infty$, we have $\mathcal{C}^{\gamma}(I) \hookrightarrow \operatorname{Bes}(\gamma, p)(I)$.

- For $1 \leqslant p<\infty$ and $\varepsilon>0$, we get $\mathcal{C}^{\gamma+\varepsilon}(I) \hookrightarrow \operatorname{bes}(\gamma, p)(I)$.

- For $1<p<\infty$ and $1 / p<\gamma<1$, we obtain $\operatorname{Bes}(\gamma, p)(I) \hookrightarrow \mathcal{C}^{\gamma-1 / p}(I)$.

- For $p \in[1, \infty)$ and $0<\gamma<1$,

$$
\operatorname{Bes}\left(\gamma, M_{2}\right)(I) \hookrightarrow \operatorname{Bes}(\gamma, p)(I) \quad \text { and } \quad \operatorname{bes}\left(\gamma, M_{2}\right)(I) \hookrightarrow \operatorname{bes}(\gamma, p)(I)
$$

In the following, we restrict ourselves to the interval $I=[0,1]$, so we will omit $I$ in our notations, e.g. $\operatorname{Bes}(\gamma, p):=\operatorname{Bes}(\gamma, p)(I)$.

\section{BESOV REGULARITY OF THE BIFRACTIONAL BROWNIAN MOTION}

Our main result is the following theorem:

THEOREM 3.1. For each $\alpha \in(0,1), \beta \in(0,1]$ and $\frac{1}{\alpha \beta}<p<\infty$, we have

$$
\mathbb{P}\left(B^{\alpha, \beta}(\cdot) \in \operatorname{Bes}(\alpha \beta, p)\right)=1 \quad \text { and } \quad \mathbb{P}\left(B^{\alpha, \beta}(\cdot) \in \operatorname{bes}(\alpha \beta, p)\right)=0,
$$

where $B^{\alpha, \beta}(\cdot)$ are the sample paths $[0,1] \ni t \mapsto B^{\alpha, \beta}(t)$.

To prove this theorem, we adapt the techniques of [9]. Let us first give some preliminary results.

The lemma below is a useful tool to obtain precise estimations in the calculations of this paper. For the proof we refer to [9].

LEMMA 3.2. Let $(X, Y)$ be a mean zero Gaussian vector such that $\mathbb{E}\left(X^{2}\right)=$ $\mathbb{E}\left(Y^{2}\right)=1$ and $\rho=|\mathbb{E} X Y|$. Then for any measurable functions $f$ and $g$ such that $\mathbb{E}(f(X))^{2}<\infty, \mathbb{E}(f(Y))^{2}<\infty$ and $f(X), f(Y)$ are centred, we have

$$
|\mathbb{E} f(X) g(Y)| \leqslant \rho\left\{\mathbb{E}(f(X))^{2}\right\}^{1 / 2}\left\{\mathbb{E}(g(Y))^{2}\right\}^{1 / 2}
$$

when $f($ or $g)$ is even we can replace $\rho$ by $\rho^{2}$ in the previous inequality.

We define

$$
u_{j k}:=2 \cdot 2^{j / 2}\left\{B^{\alpha, \beta}\left(\frac{2 k-1}{2^{j+1}}\right)-\frac{1}{2} B^{\alpha, \beta}\left(\frac{2 k}{2^{j+1}}\right)-\frac{1}{2} B^{\alpha, \beta}\left(\frac{2 k-2}{2^{j+1}}\right)\right\} .
$$

We set

$$
v_{j k}:=\frac{u_{j k}}{\sigma_{j k}} \quad \text { with } \quad \sigma_{j k}:=\left\{\mathbb{E}\left[\left|u_{j k}\right|^{2}\right]\right\}^{1 / 2}
$$


By using (1.1) and (3.1) we have, for all $j \geqslant 1$ and $k, k^{\prime} \in\left\{1, \ldots, 2^{j}\right\}$,

$$
\mathbb{E}\left[u_{j k} u_{j k^{\prime}}\right]=\frac{2^{j(1-2 \alpha \beta)}}{2^{\beta+2 \alpha \beta}}\left(\Delta_{y}^{2} \Delta_{x}^{2} \Psi_{k, k^{\prime}}(0,0)-\Delta^{4} \Phi_{k, k^{\prime}}(0)\right),
$$

where $\Delta_{y}^{2}$ (resp. $\Delta_{x}^{2}$ ) is the one step progressive difference of order 2 in the $y$ variable (resp. $x$ variable), and $\Delta^{4}$ is the one step progressive difference of order 4 , i.e.

$$
\Delta^{4} f(0)=f(4)-4 f(3)+6 f(2)-4 f(1)+f(0),
$$

and

$$
\begin{aligned}
\Delta_{y}^{2} \Delta_{x}^{2} g(0,0)= & g(2,2)-2 g(2,1)+g(2,0)-2 g(1,2)+4 g(1,1) \\
& -2 g(1,0)+g(0,2)-2 g(0,1)+g(0,0) .
\end{aligned}
$$

The functions in (3.3) are

$$
\begin{aligned}
\Psi_{k, k^{\prime}}(x, y) & :=\left((2 k-2+x)^{2 \alpha}+\left(2 k^{\prime}-2+y\right)^{2 \alpha}\right)^{\beta}, \\
\Phi_{k, k^{\prime}}(x) & :=\left|2\left(k-k^{\prime}\right)-2+x\right|^{2 \alpha \beta} .
\end{aligned}
$$

LEMMA 3.3. For all $\alpha \in(0,1), \beta \in(0,1], j \geqslant 1$ and $k, k^{\prime} \in\left\{1, \ldots, 2^{j}\right\}$ with $k^{\prime}<k$, there exist $C>0, \kappa_{k, k^{\prime}} \in(0,2)$ and $c_{k, k^{\prime}} \in(0,4)$ such that

$$
\begin{aligned}
\left|\mathbb{E}\left[u_{j k} u_{j k^{\prime}}\right]\right| \leqslant C 2^{j(1-2 \alpha \beta)}\left\{\frac{1}{\left(2 k^{\prime}-2+\kappa_{k, k^{\prime}}\right)^{4-2 \alpha \beta}}\right. & \\
& \left.+\frac{1}{\left(2\left(k-k^{\prime}\right)-2+c_{k, k^{\prime}}\right)^{4-2 \alpha \beta}}\right\} .
\end{aligned}
$$

Moreover, there exist constants $m_{1}, m_{2}>0$ such that, for all $j \geqslant 1$ and $k \in$ $\left\{1, \ldots, 2^{j}\right\}$,

$$
m_{1} 2^{j(1-2 \alpha \beta)} \leqslant \mathbb{E}\left[\left|u_{j k}\right|^{2}\right] \leqslant m_{2} 2^{j(1-2 \alpha \beta)} .
$$

Proof. Denote by $\Phi_{k, k^{\prime}}^{(4)}$ the derivative of order 4 of $\Phi_{k, k^{\prime}}$. By the mean value theorem and (3.3), there exist constants $c_{1, k, k^{\prime}}, c_{2, k, k^{\prime}} \in(0,2)$ and $c_{3, k, k^{\prime}} \in(0,4)$ such that

$$
\mathbb{E}\left[u_{j k} u_{j k^{\prime}}\right]=\frac{2^{j(1-2 \alpha \beta)}}{2^{\beta+2 \alpha \beta}}\left(\partial_{y}^{2} \partial_{x}^{2} \Psi_{k, k^{\prime}}\left(c_{1, k, k^{\prime}}, c_{2, k, k^{\prime}}\right)-\Phi_{k, k^{\prime}}^{(4)}\left(c_{3, k, k^{\prime}}\right)\right),
$$

where $\partial_{y}=\frac{\partial}{\partial y}$ and $\partial_{x}=\frac{\partial}{\partial x}$. On the other hand, we find that for all $j \geqslant 1$ and 
$k, k^{\prime} \in\left\{1, \ldots, 2^{j}\right\}$,

(3.7) $\partial_{y}^{2} \partial_{x}^{2} \Psi_{k, k^{\prime}}\left(c_{1, k, k^{\prime}}, c_{2, k, k^{\prime}}\right)$

$$
\begin{aligned}
&= 4 \alpha^{2}(2 \alpha-1)^{2} \beta(\beta-1)\left(2 k-2+c_{1, k, k^{\prime}}\right)^{2 \alpha-2}\left(2 k^{\prime}-2+c_{2, k, k^{\prime}}\right)^{2 \alpha-2} \\
& \times\left(\left(2 k-2+c_{1, k, k^{\prime}}\right)^{2 \alpha}+\left(2 k^{\prime}-2+c_{2, k, k^{\prime}}\right)^{2 \alpha}\right)^{\beta-2} \\
&+8 \alpha^{3}(2 \alpha-1) \beta(\beta-1)(\beta-2)\left(2 k-2+c_{1, k, k^{\prime}}\right)^{2 \alpha-2}\left(2 k^{\prime}-2+c_{2, k, k^{\prime}}\right)^{4 \alpha-2} \\
& \times\left(\left(2 k-2+c_{1, k, k^{\prime}}\right)^{2 \alpha}+\left(2 k^{\prime}-2+c_{2, k, k^{\prime}}\right)^{2 \alpha}\right)^{\beta-3} \\
&+8 \alpha^{3}(2 \alpha-1) \beta(\beta-1)(\beta-2)\left(2 k-2+c_{1, k, k^{\prime}}\right)^{4 \alpha-2}\left(2 k^{\prime}-2+c_{2, k, k^{\prime}}\right)^{2 \alpha-2} \\
& \times\left(\left(2 k-2+c_{1, k, k^{\prime}}\right)^{2 \alpha}+\left(2 k^{\prime}-2+c_{2, k, k^{\prime}}\right)^{2 \alpha}\right)^{\beta-3} \\
&+16 \alpha^{4} \beta(\beta-1)(\beta-2)(\beta-3)\left(2 k-2+c_{1, k, k^{\prime}}\right)^{4 \alpha-2}\left(2 k^{\prime}-2+c_{2, k, k^{\prime}}\right)^{4 \alpha-2} \\
& \times\left(\left(2 k-2+c_{1, k, k^{\prime}}\right)^{2 \alpha}+\left(2 k^{\prime}-2+c_{2, k, k^{\prime}}\right)^{2 \alpha}\right)^{\beta-4} \\
&=I_{1}+I_{2}+I_{3}+I_{4} .
\end{aligned}
$$

Moreover, for all $j \geqslant 1$ and $k, k^{\prime} \in\left\{1, \ldots, 2^{j}\right\}$ such that $k^{\prime}<k$, we have

$$
\Phi_{k, k^{\prime}}^{(4)}\left(c_{3, k, k^{\prime}}\right)=\prod_{l=0}^{3}(2 \alpha \beta-l)\left(2\left(k-k^{\prime}\right)-2+c_{3, k, k^{\prime}}\right)^{2 \alpha \beta-4} .
$$

Let us first investigate the inequality (3.4). Combining (3.6)-(3.8) we get, for all $j \geqslant 1$ and $k, k^{\prime} \in\left\{1, \ldots, 2^{j}\right\}$ such that $k^{\prime}<k$,

$$
\left|\mathbb{E}\left[u_{j k} u_{j k^{\prime}}\right]\right| \leqslant \frac{2^{j(1-2 \alpha \beta)}}{2^{\beta+2 \alpha \beta}}\left\{\sum_{l=1}^{4}\left|I_{l}\right|+\frac{\prod_{l=0}^{3}|2 \alpha \beta-l|}{\left(2\left(k-k^{\prime}\right)-2+c_{3, k, k^{\prime}}\right)^{4-2 \alpha \beta}}\right\} .
$$

- Suppose $\alpha \leqslant 1 / 2$. Let $j \geqslant 1$ and $k, k^{\prime} \in\left\{1, \ldots, 2^{j}\right\}$ such that $k^{\prime}<k$. By 3.7) we have

$$
\sum_{l=1}^{4}\left|I_{l}\right| \leqslant \frac{\tilde{C}(\alpha, \beta)}{\left(2 k^{\prime}-2+c_{1, k, k^{\prime}} \wedge c_{2, k, k^{\prime}}\right)^{4-2 \alpha \beta}},
$$

where

$$
\begin{aligned}
\tilde{C}(\alpha, \beta)=2^{\beta} \alpha^{2}|\beta-1|\left\{\beta(2 \alpha-1)^{2}+2 \alpha|2 \alpha-1| \beta|\beta-2|\right. & \\
& \left.+\alpha^{2} \beta|\beta-2||\beta-3|\right\} .
\end{aligned}
$$

Combining (3.9) and 3.10), we get

$$
\begin{aligned}
&\left|\mathbb{E}\left[u_{j k} u_{j k^{\prime}}\right]\right| \leqslant \frac{2^{j(1-2 \alpha \beta)}}{2^{\beta+2 \alpha \beta}}\left\{\frac{\tilde{C}(\alpha, \beta)}{\left(2 k^{\prime}-2+c_{1, k, k^{\prime}} \wedge c_{2, k, k^{\prime}}\right)^{4-2 \alpha \beta}}\right. \\
&\left.\quad+\frac{\prod_{l=0}^{3}|2 \alpha \beta-l|}{\left(2\left(k-k^{\prime}\right)-2+c_{3, k, k^{\prime}}\right)^{4-2 \alpha \beta}}\right\} .
\end{aligned}
$$


- Suppose $\alpha>1 / 2$. Let $j \geqslant 1$ and $k, k^{\prime} \in\left\{1, \ldots, 2^{j}\right\}$ such that $k^{\prime}<k$. We remark that

$$
\begin{aligned}
I_{2}+I_{3}= & \frac{8 \alpha^{3}(2 \alpha-1) \beta(\beta-1)(\beta-2)}{\left(2 k-2+c_{1, k, k^{\prime}}\right)^{2-2 \alpha}\left(2 k^{\prime}-2+c_{2, k, k^{\prime}}\right)^{2-2 \alpha}} \\
& \times \frac{1}{\left(\left(2 k-2+c_{1, k, k^{\prime}}\right)^{2 \alpha}+\left(2 k^{\prime}-2+c_{2, k, k^{\prime}}\right)^{2 \alpha}\right)^{2-\beta}} .
\end{aligned}
$$

By the inequality $\frac{a b}{a^{2}+b^{2}} \leqslant \frac{1}{2}$, we have

$$
\begin{aligned}
\left|I_{4}\right| \leqslant & \frac{4 \alpha^{4} \beta|\beta-1||\beta-2||\beta-3|}{\left(2 k-2+c_{1, k, k^{\prime}}\right)^{2-2 \alpha}\left(2 k^{\prime}-2+c_{2, k, k^{\prime}}\right)^{2-2 \alpha}} \\
& \times \frac{1}{\left(\left(2 k-2+c_{1, k, k^{\prime}}\right)^{2 \alpha}+\left(2 k^{\prime}-2+c_{2, k, k^{\prime}}\right)^{2 \alpha}\right)^{2-\beta}} .
\end{aligned}
$$

Combining (3.7), 3.13) and 3.14, we get

$$
\sum_{l=1}^{4}\left|I_{l}\right| \leqslant \frac{\tilde{C}(\alpha, \beta)}{\left(2 k^{\prime}-2+c_{1, k, k^{\prime}} \wedge c_{2, k, k^{\prime}}\right)^{4-2 \alpha \beta}},
$$

where $\tilde{C}(\alpha, \beta)$ is given by 3.11). According to 3.9) and 3.15), we obtain

$$
\begin{aligned}
\left|\mathbb{E}\left[u_{j k} u_{j k^{\prime}}\right]\right| \leqslant \frac{2^{j(1-2 \alpha \beta)}}{2^{\beta+2 \alpha \beta}}\left\{\frac{\tilde{C}(\alpha, \beta)}{\left(2 k^{\prime}-2+c_{1, k, k^{\prime}} \wedge c_{2, k, k^{\prime}}\right)^{4-2 \alpha \beta}}\right. \\
\left.\quad+\frac{\prod_{l=0}^{3}|2 \alpha \beta-l|}{\left(2\left(k-k^{\prime}\right)-2+c_{3, k, k^{\prime}}\right)^{4-2 \alpha \beta}}\right\} .
\end{aligned}
$$

We put

$$
C=\frac{\tilde{C}(\alpha, \beta) \vee \prod_{l=0}^{3}|2 \alpha \beta-l|}{2^{\beta+2 \alpha \beta}} .
$$

Then for all $\alpha \in(0,1), \beta \in(0,1], j \geqslant 1$ and $k, k^{\prime} \in\left\{1, \ldots, 2^{j}\right\}$ such that $k^{\prime}<k$,

$$
\begin{aligned}
&\left|\mathbb{E}\left[u_{j k} u_{j k^{\prime}}\right]\right| \leqslant C 2^{j(1-2 \alpha \beta)}\left\{\frac{1}{\left(2 k^{\prime}-2+\kappa_{k, k^{\prime}}\right)^{4-2 \alpha \beta}}\right. \\
&\left.\quad+\frac{1}{\left(2\left(k-k^{\prime}\right)-2+c_{k, k^{\prime}}\right)^{4-2 \alpha \beta}}\right\},
\end{aligned}
$$

where $\kappa_{k, k^{\prime}}=c_{1, k, k^{\prime}} \wedge c_{2, k, k^{\prime}}$ and $c_{k, k^{\prime}}=c_{3, k, k^{\prime}}$. This finishes the proof of (3.4).

Now we will prove (3.5). To this end, let us start by proving the upper bound. For all $j \geqslant 1$ and $k \in\left\{1, \ldots, 2^{j}\right\}$, by (3.3) and the mean value theorem, there exist $c_{1, k}, c_{2, k} \in(0,2)$ such that

$$
\begin{aligned}
\mathbb{E}\left[\left|u_{j k}\right|^{2}\right] & =\frac{2^{j(1-2 \alpha \beta)}}{2^{\beta+2 \alpha \beta}}\left(\partial_{y}^{2} \partial_{x}^{2} \Psi_{k, k}\left(c_{1, k}, c_{2, k}\right)-\Delta^{4} \Phi_{k, k}(0)\right) \\
& =\frac{2^{j(1-2 \alpha \beta)}}{2^{\beta+2 \alpha \beta}}\left(\partial_{y}^{2} \partial_{x}^{2} \Psi_{k, k}\left(c_{1, k}, c_{2, k}\right)+8-2^{2 \alpha \beta+1}\right) .
\end{aligned}
$$


- Suppose $\alpha \leqslant 1 / 2$. By 3.7] we note that $\partial_{y}^{2} \partial_{x}^{2} \Psi_{k, k}\left(c_{1, k}, c_{2, k}\right) \leqslant 0$, so by using (3.18) we have

$$
\mathbb{E}\left[\left|u_{j k}\right|^{2}\right] \leqslant \frac{8-2^{2 \alpha \beta+1}}{2^{\beta+2 \alpha \beta}} 2^{j(1-2 \alpha \beta)} .
$$

- Suppose $\alpha>1 / 2$. By (3.7) we observe that $I_{1}, I_{4} \leqslant 0$, and by (3.13) we get, for all $j \geqslant 1$ and $k \in\left\{2, \ldots, 2^{j}\right\}$,

$$
I_{2}+I_{3} \leqslant \frac{\alpha^{3}(2 \alpha-1) \beta(\beta-1)(\beta-2)}{2^{3-2 \alpha \beta-\beta}} .
$$

So 3.18 and 3.20 entail

$$
\begin{aligned}
& \mathbb{E}\left[\left|u_{j k}\right|^{2}\right] \\
& \quad \leqslant 2^{j(1-2 \alpha \beta)}\left(\frac{\alpha^{3}(2 \alpha-1) \beta(\beta-1)(\beta-2)}{8}+\frac{8-2^{2 \alpha \beta+1}}{2^{\beta+2 \alpha \beta}}\right) .
\end{aligned}
$$

Now for $j \geqslant 1$ and $k=1$ we have, by (3.1) and (1.2),

$$
\mathbb{E}\left[\left|u_{j 1}\right|^{2}\right]=2^{j(1-2 \alpha \beta)} \frac{4 \mathbb{E}\left[B^{\alpha, \beta}(1)-\frac{1}{2} B^{\alpha, \beta}(2)\right]^{2}}{2^{2 \alpha \beta}} .
$$

Put

$$
\begin{aligned}
m_{2}=\max \left\{\frac{\alpha^{3}(2 \alpha-1) \beta(\beta-1)(\beta-2)}{8}+\frac{8-2^{2 \alpha \beta+1}}{2^{\beta+2 \alpha \beta}},\right. \\
\left.\frac{4 \mathbb{E}\left[B^{\alpha, \beta}(1)-\frac{1}{2} B^{\alpha, \beta}(2)\right]^{2}}{2^{2 \alpha \beta}}\right\} .
\end{aligned}
$$

Combining 3.21 -3.23) and 3.19) we get, for all $\alpha \in(0,1), \beta \in(0,1], j \geqslant 1$ and $k \in\left\{1, \ldots, 2^{j}\right\}$,

$$
\mathbb{E}\left[\left|u_{j k}\right|^{2}\right] \leqslant m_{2} 2^{j(1-2 \alpha \beta)} .
$$

This finishes the proof of the upper bound in 3.5 .

Let us now investigate the lower bound. From (3.1) and (1.2), it follows that for all $j \geqslant 1$ and $k \in\left\{1, \ldots, 2^{j}\right\}$,

$$
\mathbb{E}\left[\left|u_{j k}\right|^{2}\right]=2^{j(1-2 \alpha \beta)} \frac{4 C(k)}{2^{2 \alpha \beta}},
$$

where

$$
C(k)=\mathbb{E}\left[B^{\alpha, \beta}(2 k-1)-\frac{1}{2} B^{\alpha, \beta}(2 k)-\frac{1}{2} B^{\alpha, \beta}(2 k-2)\right]^{2} .
$$

We know by [1, Lemma 3.3] (see also [24]) that the process $\left(B^{\alpha, \beta}(t)\right)_{t \geqslant 0}$ is locally non-deterministic, i.e. for all $0=t_{0}<t_{1}<\cdots<t_{m}<1$ with $t_{m}-t_{1}<\delta$ and 
$\left(u_{1}, \ldots, u_{m}\right) \in \mathbb{R}^{m}$,

$$
\begin{aligned}
\operatorname{Var}\left(\sum_{j=1}^{m} u_{j}\left[B^{\alpha, \beta}\left(t_{j}\right)-B^{\alpha, \beta}\left(t_{j-1}\right)\right]\right) & \\
& \geqslant C_{m} \sum_{j=1}^{m} u_{j}^{2} \operatorname{Var}\left(B^{\alpha, \beta}\left(t_{j}\right)-B^{\alpha, \beta}\left(t_{j-1}\right)\right) .
\end{aligned}
$$

On the other hand, by (1.2) we have, for some $\varepsilon<\frac{\delta}{2} \wedge \frac{1}{2 k}$,

$$
\begin{aligned}
C(k)=\varepsilon^{-2 \alpha \beta} \mathbb{E}\left[\frac{1}{2}\left[B^{\alpha, \beta}(\varepsilon(2 k-1))-B^{\alpha, \beta}(\varepsilon(2 k-2))\right]\right. & \\
& \left.-\frac{1}{2}\left[B^{\alpha, \beta}(\varepsilon(2 k))-B^{\alpha, \beta}(\varepsilon(2 k-1))\right]\right]^{2} .
\end{aligned}
$$

Combining 3.26, 3.27) and 1.3 , we get $C(k) \geqslant \frac{C_{3}}{2^{1+\beta}}$. Hence

$$
\mathbb{E}\left[\left|u_{j k}\right|^{2}\right] \geqslant m_{1} 2^{j(1-2 \alpha \beta)} \quad \text { with } \quad m_{1}=\frac{4 C_{3}}{2^{2 \alpha \beta+\beta+1}} .
$$

This finishes the proof of Lemma 3.3

REMARK 3.4. We remark that if $\beta=1$, then $\partial_{y}^{2} \partial_{x}^{2} \Psi_{k, k^{\prime}}\left(c_{1, k, k^{\prime}}, c_{2, k, k^{\prime}}\right)=0$ and hence $\Delta_{y}^{2} \Delta_{x}^{2} \Psi_{k, k^{\prime}}(0,0)=0$, so equation (3.3) becomes

$$
\mathbb{E}\left[u_{j k} u_{j k^{\prime}}\right]=-\frac{2^{j(1-2 \alpha)}}{2^{1+2 \alpha}} \Delta^{4} \Phi_{k, k^{\prime}}(0),
$$

the same as in [9, (IV.9)] for the fractional Brownian motion.

LEMMA 3.5. There exists a constant $M>0$ such that, for all $j \geqslant 1$ and $k, k^{\prime} \in\left\{1, \ldots, 2^{j}\right\}$, we have

$$
\sum_{k, k^{\prime}=1}^{2^{j}}\left|\mathbb{E} v_{j k} v_{j k^{\prime}}\right|^{2} \leqslant M 2^{j},
$$

where the $v_{j k}$ are given by (3.2).

Proof. Equality (3.2) and Hölder's inequality give

$$
\begin{aligned}
\sum_{k, k^{\prime}=1}^{2^{j}}\left|\mathbb{E} v_{j k} v_{j k^{\prime}}\right|^{2}= & 2 \sum_{\substack{k^{\prime}<k, k^{\prime} \geqslant 2 \\
k-k^{\prime} \geqslant 2}}^{2^{j}}\left|\mathbb{E} v_{j k} v_{j k^{\prime}}\right|^{2}+2 \sum_{\substack{k^{\prime}<k, k^{\prime} \geqslant 2 \\
k-k^{\prime}=1}}^{2^{j}}\left|\mathbb{E} v_{j k} v_{j k^{\prime}}\right|^{2} \\
& +2 \sum_{\substack{k=2 \\
2^{j}}}\left|\mathbb{E} v_{j k} v_{j 1}\right|^{2}+\sum_{k=1}^{2^{j}}\left\{\mathbb{E}\left[\left|v_{j k}\right|^{2}\right]\right\}^{2} \\
\leqslant & 2 \sum_{\substack{k^{\prime}<k, k^{\prime} \geqslant 2 \\
k-k^{\prime} \geqslant 2}}^{2^{j}}\left|\frac{\mathbb{E} u_{j k} u_{j k^{\prime}}}{\sigma_{j k} \sigma_{j k^{\prime}}}\right|^{2}+2\left(2^{j}-2\right)+2\left(2^{j}-1\right)+2^{j} \\
= & 2 J+5 \cdot 2^{j}-6 .
\end{aligned}
$$


We will estimate $J$. To this end, set $A=2 C^{2} / m_{2}^{2}$. Then by 3.4 and 3.5,

$$
\begin{aligned}
& J \leqslant A \sum_{\substack{k^{\prime}<k, k^{\prime} \geqslant 2 \\
k-k^{\prime} \geqslant 2}}^{2^{j}}\left\{\frac{1}{\left(2 k^{\prime}-2+\kappa_{k, k^{\prime}}\right)^{8-4 \alpha \beta}}+\frac{1}{\left(2\left(k-k^{\prime}\right)-2+c_{k, k^{\prime}}\right)^{8-4 \alpha \beta}}\right\} \\
& =A \sum_{k=4}^{2^{j}} \sum_{k^{\prime}=2}^{k-2}\left\{\frac{1}{\left(2 k^{\prime}-2+\kappa_{k, k^{\prime}}\right)^{8-4 \alpha \beta}}+\frac{1}{\left(2\left(k-k^{\prime}\right)-2+c_{k, k^{\prime}}\right)^{8-4 \alpha \beta}}\right\} \\
& \leqslant A \sum_{k=4}^{2^{j}} \sum_{k^{\prime}=2}^{k-2}\left\{\frac{1}{\left(2 k^{\prime}-2\right)^{8-4 \alpha \beta}}+\frac{1}{\left(2\left(k-k^{\prime}\right)-2\right)^{8-4 \alpha \beta}}\right\} \\
& \leqslant A \sum_{k=4}^{2^{j}} \sum_{k^{\prime}=2}^{k-2}\left\{\int_{2 k^{\prime}-3}^{2 k^{\prime}-2} \frac{1}{x^{8-4 \alpha \beta}} d x+\int_{2\left(k-k^{\prime}\right)-3}^{2\left(k-k^{\prime}\right)-2} \frac{1}{x^{8-4 \alpha \beta}} d x\right\} \\
& =2 A \sum_{k=4}^{2^{j}} \sum_{k^{\prime}=2}^{k-2} \int_{k^{\prime}-3}^{2 k^{\prime}-2} \frac{1}{x^{8-4 \alpha \beta}} d x \leqslant 2 A \sum_{k=4}^{2^{j}} \int_{1}^{2 k-6} \frac{1}{x^{8-4 \alpha \beta}} d x \\
& \leqslant \frac{2 A}{7-4 \alpha \beta}\left(2^{j}-3\right) \text {. }
\end{aligned}
$$

This proves Lemma 3.5

LEMMA 3.6. For all $j \geqslant 1$ and $k \in\left\{1, \ldots, 2^{j}\right\}$, we have

$$
\mathbb{E}\left[\sum_{k=1}^{2^{j}}\left(\left|v_{j k}\right|^{p}-c_{p}\right)\right]^{2} \leqslant\left(c_{2 p}-c_{p}^{2}\right) M 2^{j},
$$

where $c_{p}=\frac{1}{\sqrt{2 \pi}} \int_{\mathbb{R}}|x|^{p} e^{-x^{2} / 2} d x$.

Proof. First we get

$$
\mathbb{E}\left[\sum_{k=1}^{2^{j}}\left(\left|v_{j k}\right|^{p}-c_{p}\right)\right]^{2}=\sum_{k, k^{\prime}=1}^{2^{j}} \mathbb{E}\left[\left(\left|v_{j k}\right|^{p}-c_{p}\right)\left(\left|v_{j k^{\prime}}\right|^{p}-c_{p}\right)\right] .
$$

By applying Lemma 3.2 with $f(x)=g(x)=|x|^{p}-c_{p}$, we obtain

$$
\mathbb{E}\left[\sum_{k=1}^{2^{j}}\left(\left|v_{j k}\right|^{p}-c_{p}\right)\right]^{2} \leqslant\left(c_{2 p}-c_{p}^{2}\right) \sum_{k, k^{\prime}=1}^{2^{j}}\left|\mathbb{E}\left[v_{j k} v_{j k^{\prime}}\right]\right|^{2} .
$$

Inequality 3.29) of Lemma 3.5 ends the proof.

Proof of Theorem 3.1. We are going to prove that, almost surely,

$$
2^{-j} \sum_{k=1}^{2^{j}}\left|v_{j k}\right|^{p} \underset{j \rightarrow \infty}{\longrightarrow} c_{p}
$$

To this end we will show that for all $\varepsilon>0$,

$$
\sum_{j \geqslant 1} \mathbb{P}\left\{2^{-j} \sum_{k=1}^{2^{j}}\left|v_{j k}\right|^{p} \notin\left[c_{p}-\varepsilon, c_{p}+\varepsilon\right]\right\}<\infty .
$$


Markov's inequality gives

$$
\mathbb{P}\left\{2^{-j} \sum_{k=1}^{2^{j}}\left|v_{j k}\right|^{p} \notin\left[c_{p}-\varepsilon, c_{p}+\varepsilon\right]\right\} \leqslant \frac{1}{\varepsilon^{2} 2^{2 j}} \mathbb{E}\left[\sum_{k=1}^{2^{j}}\left(\left|v_{j k}\right|^{p}-c_{p}\right)\right]^{2} .
$$

Combining (3.33) and Lemma 3.6, we find that 3.32 holds, and 3.31) is then a consequence of the Borel-Cantelli Lemma. Finally, Theorem 3.1 is a simple consequence of Theorem 2.1 .

Here is a stronger regularity result than Theorem 3.1 .

THEOREM 3.7. For each $\alpha \in(0,1)$ and $\beta \in(0,1]$, we have

$$
\mathbb{P}\left(B^{\alpha, \beta}(\cdot) \in \operatorname{Bes}\left(\alpha \beta, M_{2}\right)\right)=1 \quad \text { and } \quad \mathbb{P}\left(B^{\alpha, \beta}(\cdot) \in \operatorname{bes}\left(\alpha \beta, M_{2}\right)\right)=0,
$$

where $B^{\alpha, \beta}(\cdot)$ are the sample paths $[0,1] \ni t \mapsto B^{\alpha, \beta}(t)$.

Proof. The proof is similar to that of [9, Theorem II.5]. Indeed, taking into account Theorem 2.2, Lemma 3.6, and the fact that for positive integers $p$, we have

$$
c_{2 p}=(2 p) ! /\left(p ! 2^{p}\right) \underset{p \rightarrow \infty}{\sim} e^{-p} 2^{p+1 / 2} p^{p},
$$

we infer that there exists a constant $c>1$ such that $c_{2 p} \leqslant c e^{-p}(2 p)^{p}$.

REMARK 3.8. (1) Let $\left(Y_{t}^{\alpha}\right)_{t \geqslant 0}$ be a sub-fractional Brownian motion, i.e. a mean zero Gaussian process with covariance function

$$
\mathbb{E}\left[Y_{t}^{\alpha} Y_{s}^{\alpha}\right]=s^{2 \alpha}+t^{2 \alpha}-\frac{1}{2}\left[(s+t)^{2 \alpha}+|t-s|^{2 \alpha}\right],
$$

where $\alpha \in(0,1)$. We believe that by the same calculations as above one can deduce that almost all paths of the sub-fractional Brownian motion belong to (resp. do not belong to) the $\operatorname{Besov}$ space $\operatorname{Bes}(\alpha, p)$ and the $\operatorname{Besov-Orlicz}$ space $\operatorname{Bes}\left(\alpha, M_{2}\right)$ (resp. $\operatorname{bes}(\alpha, p)$ and bes $\left(\alpha, M_{2}\right)$ ).

(2) Let $r(t)$ be a real valued function such that the kernel $K_{r}(t, s)$ defined by

$$
K_{r}(t, s):=r(t)-r(s)-r(t-s)
$$

is positive on the real line, and let

$$
\varphi(t)=\int_{0}^{\infty}\left(1-e^{-u|t|}\right) d m(u)
$$

where $m$ is a positive measure on $[0, \infty)$ such that $\int_{1}^{\infty} d m(u)<\infty$. Therefore by [2, Theorem 5.1],

$$
K(s, t):=\varphi(r(t)+r(s))-\varphi(r(t-s))
$$

is a positive kernel on the real line. Let $\left(X_{t}\right)_{t \geqslant 0}$ be a centred Gaussian process with covariance function

$$
\mathbb{E}\left[X_{t} X_{s}\right]=K(s, t)
$$


If in addition we assume that $\varphi$ and $r$ are in $\mathcal{C}^{4}((0, \infty))$, and that for all $a>0$, we have $r(a t)=a^{\alpha} r(t)$ and $\varphi(a x)=a^{\beta} \varphi(x)$ (i.e. $X$ is a self-similar Gaussian process with index $\alpha \beta / 2$ ), then we expect that almost all paths of the proces $X$ belong to (resp. do not belong to) the $\operatorname{Besov}$ spaces $\operatorname{Bes}(\alpha \beta / 2, p)$ (resp. bes $(\alpha \beta / 2, p)$ ). We intend to provide, in a future paper, a proof of this result using a new method dealing directly with Besov norms without using Ciesielski's isomorphism theorem. For this new approach, we refer to [15], [31], [23].

(3) The bifractional Brownian motion $B^{\alpha, \beta}$ with $0<\alpha<1,1<\beta<2$ such that $\alpha \beta<1$ has been introduced and studied in [3]. The case $\alpha>1$ such that $2 \alpha \beta \leqslant 1$ has been considered in [28]. One can show that the regularity results in Theorems 3.1 and 3.7 remain valid here, since the derivatives $\partial_{y}^{2} \partial_{x}^{2} \Psi_{k, k^{\prime}}\left(c_{1, k, k^{\prime}}, c_{2, k, k^{\prime}}\right)$ and $\Phi_{k, k^{\prime}}^{(4)}\left(c_{3, k, k^{\prime}}\right)$ do not change. So in the proof of Lemma 3.3, we need just to take into account the other cases (i.e. $0<\alpha<1$, $1<\beta<2$ such that $\alpha \beta<1$ and $\alpha>1$ with $2 \alpha \beta \leqslant 1$ ).

\section{AN ITÔ-NISIO THEOREM FOR THE BIFRACTIONAL BROWNIAN MOTION}

Let $\mathcal{E}$ be the linear space generated by the indicator functions $\mathbb{1}_{[0, t]}$ endowed with the inner product

$$
\left\langle\mathbb{1}_{[0, t]}, \mathbb{1}_{[0, s]}\right\rangle_{\mathcal{H}}=R(s, t), \quad s, t \in[0,1] .
$$

Define $\mathcal{H}$ as the completion of $\mathcal{E}$ with respect to the inner product $\langle\cdot, \cdot\rangle_{\mathcal{H}}$. The map $\mathcal{H} \ni \varphi \mapsto B^{\alpha, \beta}(\varphi)$ is an isometry from $\mathcal{H}$ to the Gaussian space generated by $B^{\alpha, \beta}$. Moreover, $B^{\alpha, \beta}(\varphi)$ is the Wiener integral of $\varphi$ with respect to $B^{\alpha, \beta}$.

Our main result in this section is the following Itô-Nisio theorem for the bifractional Brownian motion.

THEOREM 4.1. Suppose $\alpha \beta>1 / 2$ and let $\left(\varphi_{n}\right)_{n \geqslant 1}$ be an orthonormal basis of $\mathcal{H}$. Then almost surely

$\sum_{n=1}^{N}\left\langle\varphi_{n}, \mathbb{1}_{[0, t]}\right\rangle_{\mathcal{H}} B^{\alpha, \beta}\left(\varphi_{n}\right) \underset{N \rightarrow \infty}{\longrightarrow} B^{\alpha, \beta}(t) \quad$ in the Besov space $\operatorname{Bes}(\alpha \beta-\varepsilon, p)$,

where $\varepsilon>0$ and $p \geqslant 1$ are such that $1 / 2<\alpha \beta-\varepsilon-1 / p$.

By classical continuous injections we can deduce

Corollary 4.2. Suppose $\alpha \beta>1 / 2$ and let $\left(\varphi_{n}\right)_{n \geqslant 1}$ be an orthonormal basis of $\mathcal{H}$. Then almost surely

$$
\sum_{n=1}^{N}\left\langle\varphi_{n}, \mathbb{1}_{[0, t]}\right\rangle_{\mathcal{H}} B^{\alpha, \beta}\left(\varphi_{n}\right) \underset{N \rightarrow \infty}{\longrightarrow} B^{\alpha, \beta}(t) \quad \text { in the Hölder space } \mathcal{C}^{\gamma}
$$

for any $\gamma<\alpha \beta$. 
Proof of Theorem 4.1. Put $X_{N}(t):=\sum_{n=1}^{N}\left\langle\varphi_{n}, \mathbb{1}_{[0, t]}\right\rangle_{\mathcal{H}} B^{\alpha, \beta}\left(\varphi_{n}\right)$ and define

$$
z_{j k}:=2 \cdot 2^{j / 2}\left\{X_{N}\left(\frac{2 k-1}{2^{j+1}}\right)-\frac{1}{2} X_{N}\left(\frac{2 k}{2^{j+1}}\right)-\frac{1}{2} X_{N}\left(\frac{2 k-2}{2^{j+1}}\right)\right\} .
$$

Let $\left\{h_{j k} ; j \geqslant 0, k=1, \ldots, 2^{j}\right\}$ be the Haar functions defined by

$$
h_{j k}=\sqrt{2^{j}} \mathbb{1}_{\left[\frac{2 k-2}{2^{j}+1}, \frac{2 k-1}{2^{j+1}}\right)}-\sqrt{2^{j}} \mathbb{1}_{\left[\frac{2 k-1}{2^{j+1}}, \frac{2 k}{2^{j+1}}\right)} \quad \text { and } \quad h_{1}=\mathbb{1}_{[0,1]} .
$$

Note that

$$
\begin{aligned}
u_{j k}-z_{j k} & =B^{\alpha, \beta}\left(h_{j k}\right)-X_{N}\left(h_{j k}\right) \\
& =B^{\alpha, \beta}\left(h_{j k}\right)-\sum_{n=1}^{N}\left\langle\varphi_{n}, h_{j k}\right\rangle_{\mathcal{H}} B^{\alpha, \beta}\left(\varphi_{n}\right) .
\end{aligned}
$$

Set

$$
\omega_{j k}^{N}=\frac{u_{j k}-z_{j k}}{\varrho_{j k}^{N}} \quad \text { with } \quad \varrho_{j k}^{N}=\left\{\mathbb{E}\left[\left|u_{j k}-z_{j k}\right|^{2}\right]\right\}^{1 / 2} .
$$

First, by the Borel-Cantelli lemma, we can easily show that almost surely

$$
2^{-j(1+\varepsilon p)} \sum_{k=1}^{2^{j}}\left|\omega_{j k}^{N}\right|^{p} \underset{j \rightarrow \infty}{\longrightarrow} 0 .
$$

Let $\phi, \psi \in \mathcal{H}$ and let $\sum_{i=1}^{L_{n}} \lambda_{i}^{n} \mathbb{1}_{\left[0, t_{i}^{n}\right]}, \sum_{j=1}^{M_{m}} \mu_{j}^{m} \mathbb{1}_{\left[0, s_{j}^{m}\right]}$ be two sequences in $\mathcal{E}$ such that

$$
\phi=\lim _{n \rightarrow \infty} \sum_{i=1}^{L_{n}} \lambda_{i}^{n} \mathbb{1}_{\left[0, t_{i}^{n}\right]} \quad \text { and } \quad \psi=\lim _{m \rightarrow \infty} \sum_{j=1}^{M_{m}} \mu_{j}^{m} \mathbb{1}_{\left[0, s_{j}^{m}\right]} \quad \text { in } \mathcal{H} .
$$

For all $a>0$, define

$$
\phi^{a}=\lim _{n \rightarrow \infty} \sum_{i=1}^{L_{n}} \lambda_{i}^{n} \mathbb{1}_{\left[0, a t_{i}^{n}\right]} \quad \text { and } \quad \psi^{a}=\lim _{m \rightarrow \infty} \sum_{j=1}^{M_{m}} \mu_{j}^{m} \mathbb{1}_{\left[0, a s_{j}^{m}\right]} \quad \text { in } \mathcal{H} .
$$

One can easily see by (1.2) and a density argument that

$$
\mathbb{E}\left[B^{\alpha, \beta}\left(\phi^{a}\right) B^{\alpha, \beta}\left(\psi^{a}\right)\right]=a^{2 \alpha \beta} \mathbb{E}\left[B^{\alpha, \beta}(\phi) B^{\alpha, \beta}(\psi)\right] .
$$

Setting $\theta_{j, k}^{n}=\left\langle\varphi_{n}, h_{j k}\right\rangle_{\mathcal{H}}$, we get

$$
\begin{aligned}
\left|\varrho_{j k}^{N}\right|^{2} & =\mathbb{E}\left[B^{\alpha, \beta}\left(h_{j k}\right)-\sum_{n=1}^{N}\left\langle\varphi_{n}, h_{j k}\right\rangle_{\mathcal{H}} B^{\alpha, \beta}\left(\varphi_{n}\right)\right]^{2} \\
& =\left\|h_{j k}\right\|_{\mathcal{H}}^{2}+\sum_{n=1}^{N}\left|\theta_{j, k}^{n}\right|^{2}-2 \sum_{n=1}^{N}\left|\theta_{j, k}^{n}\right|^{2}=\sum_{n=N+1}^{\infty}\left|\theta_{j, k}^{n}\right|^{2} .
\end{aligned}
$$


Put

$$
\begin{aligned}
\tilde{h}_{j k} & =\mathbb{1}_{\left[\frac{2 k-2}{2^{j+1}}, \frac{2 k-1}{2^{j+1}}\right)}-\mathbb{1}_{\left[\frac{2 k-1}{2^{j+1}}, \frac{2 k}{2^{j+1}}\right)}, \\
\tilde{g}_{j k} & =\mathbb{1}_{\left[\frac{2 k-2}{2^{(j+1) / 2}}, \frac{2 k-1}{2^{(j+1) / 2}}\right)}-\mathbb{1}_{\left[\frac{2 k-1}{2^{(j+1) / 2}}, \frac{2 k}{2^{(j+1) / 2}}\right)}, \\
g_{k} & =\mathbb{1}_{[2 k-2,2 k-1)}-\mathbb{1}_{[2 k-1,2 k)} .
\end{aligned}
$$

Letting $a_{j}=2^{-(j+1) / 2}$, by (4.4) we have

$$
\begin{aligned}
\theta_{j, k}^{n} & =\mathbb{E}\left[B^{\alpha, \beta}\left(h_{j k}\right) B^{\alpha, \beta}\left(\varphi_{n}\right)\right]=2^{j / 2} \mathbb{E}\left[B^{\alpha, \beta}\left(\tilde{h}_{j k}\right) B^{\alpha, \beta}\left(\varphi_{n}\right)\right] \\
& =2^{j / 2} \mathbb{E}\left[B^{\alpha, \beta}\left(\tilde{g}_{j k}^{a_{j}}\right) B^{\alpha, \beta}\left(\left(\varphi_{n}^{a_{j}^{-1}}\right)^{a_{j}}\right)\right] \\
& =2^{-\alpha \beta} 2^{j(1 / 2-\alpha \beta)} \mathbb{E}\left[B^{\alpha, \beta}\left(\tilde{g}_{j k}\right) B^{\alpha, \beta}\left(\varphi_{n}^{a_{j}^{-1}}\right)\right] .
\end{aligned}
$$

Hence combining (4.5) and 4.7), we get

$$
\begin{aligned}
\left|\varrho_{j k}^{N}\right|^{2} & =2^{-2 \alpha \beta} 2^{j(1-2 \alpha \beta)} \sum_{n=N+1}^{\infty}\left\{\mathbb{E}\left[B^{\alpha, \beta}\left(\tilde{g}_{j k}\right) B^{\alpha, \beta}\left(\varphi_{n}^{a_{j}^{-1}}\right)\right]\right\}^{2} \\
& \leqslant 2^{-2 \alpha \beta} 2^{j(1-2 \alpha \beta)} \sup _{j, k} \sum_{n=N+1}^{\infty}\left\{\mathbb{E}\left[B^{\alpha, \beta}\left(\tilde{g}_{j k}\right) B^{\alpha, \beta}\left(\varphi_{n}^{a_{j}^{-1}}\right)\right]\right\}^{2} .
\end{aligned}
$$

The last supremum is finite. In fact, we remark that $\left(a_{j}^{\alpha \beta} \varphi_{n}^{a_{j}^{-1}}\right)_{n \geqslant 1}$ is an orthonormal basis of $\mathcal{H}$, and therefore

$$
\begin{aligned}
& \sum_{n=N+1}^{\infty}\left\{\mathbb{E}\left[B^{\alpha, \beta}\left(\tilde{g}_{j k}\right) B^{\alpha, \beta}\left(\varphi_{n}^{a_{j}^{-1}}\right)\right]\right\}^{2}=a_{j}^{-2 \alpha \beta} \sum_{n=N+1}^{\infty}\left\langle\tilde{g}_{j k}, a_{j}^{\alpha \beta} \varphi_{n}^{a_{j}^{-1}}\right\rangle_{\mathcal{H}}^{2} \\
\leqslant & a_{j}^{-2 \alpha \beta} \sum_{n=1}^{\infty}\left\langle\tilde{g}_{j k}, a_{j}^{\alpha \beta} \varphi_{n}^{a_{j}^{-1}}\right\rangle_{\mathcal{H}}^{2}=a_{j}^{-2 \alpha \beta}\left\|\tilde{g}_{j k}\right\|_{\mathcal{H}}^{2}=a_{j}^{-2 \alpha \beta}\left\|g_{k}^{a_{j}}\right\|_{\mathcal{H}}^{2}=\left\|g_{k}\right\|_{\mathcal{H}}^{2} \cdot
\end{aligned}
$$

On the other hand, by $(3.24)$ we have $\mathbb{E}\left[\left|u_{j k}\right|^{2}\right] \leqslant m_{2} 2^{j(1-2 \alpha \beta)}$. Therefore by (3.1), (1.2), (4.6) and (4.1) we get, for all $k \geqslant 1$,

$$
\left\|g_{k}\right\|_{\mathcal{H}}^{2}=\mathbb{E}\left[2 B^{\alpha, \beta}(2 k-1)-B^{\alpha, \beta}(2 k)-B^{\alpha, \beta}(2 k-2)\right]^{2} \leqslant m_{2} .
$$

According to (4.9), for all $N \geqslant 1$ we obtain

$$
A_{N}:=\sup _{j, k} \sum_{n=N+1}^{\infty}\left\{\mathbb{E}\left[B^{\alpha, \beta}\left(\tilde{g}_{j k}\right) B^{\alpha, \beta}\left(\varphi_{n}^{a_{j}^{-1}}\right)\right]\right\}^{2}<\infty .
$$

Then $\left(A_{N}\right)_{N \geqslant 1}$ is a non-increasing real valued sequence such that

$$
\lim _{N} A_{N}=0 \quad \text { a.s. }
$$


From 4.2, 4.8) and 4.11) we derive

$$
2^{-j(1+\varepsilon p)} \sum_{k=1}^{2^{j}}\left|\omega_{j k}^{N}\right|^{p} \geqslant 2^{p \alpha \beta} 2^{-j p(1 / 2-(\alpha \beta-\varepsilon)+1 / p)} \sum_{k=1}^{2^{j}} \frac{\left|u_{j k}-z_{j k}\right|^{p}}{A_{N}^{p / 2}} .
$$

We remark that the sequence $2^{-j p(1 / 2-(\alpha \beta-\varepsilon)+1 / p)} \sum_{k=1}^{2^{j}}\left|u_{j k}-z_{j k}\right|^{p}$ is increasing in $j$ for $\varepsilon$ small enough, $p$ large enough and $\alpha \beta>1 / 2$. Therefore by (4.3) and 4.13, almost surely,

$$
\sup _{j \geqslant 0} 2^{-j p(1 / 2-(\alpha \beta-\varepsilon)+1 / p)} \sum_{k=1}^{2^{j}}\left|u_{j k}-z_{j k}\right|^{p} \leqslant 2^{-p \alpha \beta} A_{N}^{p / 2},
$$

which finishes the proof of Theorem 4.1 by applying (4.12) and Theorem 2.1.

REMARK 4.3. When $\beta=1$ we get the Itô-Nisio theorem for the fractional Brownian motion with $\alpha>1 / 2$.

Acknowledgments. We would like to warmly thank the referees for their careful reading and relevant comments. Their suggestions concerning the regularity in Besov-Orlicz space (Theorem 3.7) and the discussion of the regularity of the $\mathrm{bBm}$ in the case $\alpha>1$ (Remark 3.8) helped us to improve the quality of this paper.

\section{REFERENCES}

[1] M. Ait Ouahra, H. Ouahhabi and A. Sghir, Continuity in law of some additive functionals of the bifractional Brownian motion, Stochastics 91 (2019), 613-628.

[2] D. Alpay and D. Levanony, On the reproducing kernel Hilbert spaces associated with the fractional and bi-fractional Brownian motions, Potential Anal. 28 (2008), 163-184.

[3] X. Bardina and K. Es-Sebaiy, An extension of bifractional Brownian motion. Comm. Stoch. Anal. 5 (2011), 333-340.

[4] T. Bojdecki, L. G. Gorostiza and A. Talarczyk, Some extensions of fractional Brownian motion and sub-fractional Brownian motion related to particle systems, Electron. Comm. Probab. 12 (2007), 161-172.

[5] B. Boufoussi, E. Lakhel and M. Dozzi, A Kolmogorov and tightness criterion in modular Besov spaces and an application to a class of Gaussian processes, Stoch. Anal. Appl. 23 (2005), 665685.

[6] B. Boufoussi and Y. Nachit, Besov regularity and local time of the solution to stochastic heat equation, arXiv:2006.04235v1 (2020).

[7] Z. Ciesielski, Modulus of smoothness of the Brownian paths in the $L^{p}$ norm, in: Constructive Theory of Functions (Varna, 1991), 71-75.

[8] Z. Ciesielski, Orlicz spaces, spline systems and Brownian motion, Constr. Approx. 9 (1993), 191-208.

[9] Z. Ciesielski, G. Keryacharian and B. Roynette, Quelques espaces fonctionnels associés à des processus gaussiens, Studia Math. 107 (1993), 171-204.

[10] C. El-Nouty, The increments of a bifractional Brownian motion, Studia Sci. Math. Hungar. 46 (2009), 449-478.

[11] C. El-Nouty and J. L. Journé, Upper classes of the bifractional Brownian motion, Studia Sci. Math. Hungar. 48 (2011), 371-407. 
[12] K. Es-Sebaiy and C. A. Tudor, Multidimensional bifractional Brownian motion: Itô and Tanaka's formulas, Stoch. Dynam. 3 (2007), 365-388.

[13] X. Fernique, Régularité de processus gaussiens, Invent. Math. 12 (1971), 304-320.

[14] C. Houdré and J. Villa, An example of infinite dimensional quasi-helix, in: Stochastic Models, Contemp. Math. 366, Amer. Math. Soc., 2003, 195-201.

[15] T. P. Hytönen and M.C. Veraar, On Besov regularity of Brownian motions in infinite dimensions, Probab. Math. Statist. 28 (2008), 143-162.

[16] K. Itô and M. Nisio, On the convergence of sums of independent Banach space valued random variables, Osaka J. Math. 5 (1968), 35-48.

[17] J.-P. Kahane, Hélices et quasi-hélices, Adv. Math. 7B (1981), 417-433.

[18] J.-P. Kahane, Some Random Series of Functions, Cambridge Univ. Press, 1985.

[19] G. Kerkyacharian and B. Roynette, Une démonstration simples des théorèmes de Kolmogorov, Donsker et Ito-Nisio, C. R. Acad. Sci. Paris Sér. 312 (1991), 877-882.

[20] I. Kruk, F. Russo and C. A. Tudor, Wiener integrals, Malliavin calculus and covariance structure measure, J. Funct. Anal. 249 (2007), 92--142.

[21] H. Lakhel, Y. Ouknine and C. A. Tudor, Besov regularity for the indefinite Skorohod integral with respect to the fractional Brownian motion: the singular case, Stoch. Stoch. Reports 74 (2002), 597-615.

[22] P. Lei and D. Nualart, A decomposition of the bi-fractional Brownian motion and some applications, Statist. Probab. Lett. 79 (2009), 619-624.

[23] M. Ondreját and M. Veraar, On temporal regularity of stochastic convolutions in 2-smooth Banach spaces, Ann. Inst. H. Poincaré Probab. Statist. 56(3) (2020), 1792-1808.

[24] I. Mendy, On the local time of sub-fractional Brownian motion, Ann. Math. Blaise Pascal 17 (2010), 357-374.

[25] D. Nualart and Y. Ouknine, Besov regularity of stochastic integrals with respect to the fractional Brownian motion with parameter $H>1 / 2$, J. Theoret. Probab. 16 (2003), 451-470.

[26] B. Roynette, Mouvement Brownien et espaces de Besov, Stoch. Stoch. Reports 43 (1993), $221-$ 260.

[27] F. Russo and C. A. Tudor, On the bifractional Brownian motion, Stoch. Process. Appl. 5 (2006), 830-856.

[28] A. Talarczyk, Bifractional Brownian motion for $H>1$ and $2 H K \leqslant 1$, Statist. Probab. Lett. 157 (2020), art. 108628, 6 pp.

[29] H. Triebel, Interpolation Theory, Function Spaces, Differential Operators, 2nd ed., Johann Ambrosius Barth, Heidelberg, 1995.

[30] C. A. Tudor and Y. Xiao, Sample path properties of bifractional Brownian motion, Bernoulli 13 (2007), 1023-1052.

[31] M. C. Veraar, Correlation inequalities and applications to vector-valued Gaussian random variables and fractional Brownian motion, Potential Anal. 30 (2009), 341-370.

Brahim Boufoussi, Yassine Nachit

Department of Mathematics

Faculty of Sciences Semlalia

Cadi Ayyad University

2390 Marrakesh, Morocco

E-mail: boufoussi@uca.ac.ma

yassine.nachit.fssm@gmail.com

Received 11.7.2020;

revised version 1.12 .2020 